\title{
Cumulative beam breakup in linear accelerators with time-dependent parameters
}

\author{
J. R. Delayen* \\ Thomas Jefferson National Accelerator Facility, Newport News, Virginia 23606, USA
}

(Received 18 October 2004; published 8 February 2005)

\begin{abstract}
A formalism presented in a previous paper for the analysis of cumulative beam breakup (BBU) with arbitrary time dependence of the beam current and with misalignment of the cavities and focusing elements [J. R. Delayen, Phys. Rev. ST Accel. Beams 6, 084402 (2003)] is extended to include time dependence of the focusing and coupling between the beam and the dipole modes. Such time dependence, which could result from an energy chirp imposed on the beam or from rf focusing, is known to be effective in reducing BBU-induced instabilities and emittance growth. The analytical results are presented and applied to practical accelerator configurations and compared to numerical simulations.
\end{abstract}

DOI: 10.1103/PhysRevSTAB.8.024402

PACS numbers: 29.27.Bd, 41.75.-i, 52.35.-g

\section{INTRODUCTION}

The cumulative beam breakup instability (BBU) in linear accelerators results when a beam is injected into an accelerator with a lateral offset or an angular divergence and couples to the dipole modes of the accelerating structures [1]. The dipole modes that are excited in a cavity by the previous bunches can further deflect the following bunches and thereby increase the excitation of the dipole modes in the downstream cavities. In the case of bunches of finite length, a particle will also experience the wakefield generated by all the particles ahead of it within the same bunch. In this process the transverse displacement can be amplified and lead to a degradation of beam quality and possibly beam loss. This instability is cumulative since the transverse deflection of a particular bunch or particle results from the additive contributions from all the previous particles whether in the same bunch or in previous bunches.

Cumulative BBU has been studied in the past mostly in the context of high energy electron accelerators where the beam current profiles were comprised of periodic trains of pointlike bunches [2-8] or for high-current quasi-dc beams [9-13]. Growing interest in high-current superconducting ion accelerators for spallation sources, where the bunches have a finite length, motivated an investigation of cumulative BBU in linear accelerators with periodic beam current profile [14]. BBU in recirculating and energy recovering linacs is also very much of interest but is not addressed here; studies of multipass BBU to date have been limited to beams comprised of pointlike bunches $[15,16]$.

More recently a general analysis of BBU with arbitrary time dependence of the beam current and injection offsets, as well as random displacement of cavities and focusing elements, was developed $[17,18]$. This analysis is extended here to include time dependence of the focusing and of the coupling between the beam and the dipole mode in order to

*Electronic address: delayen@jlab.org control or suppress the BBU instability and the resulting emittance growth. This can be accomplished either with $\mathrm{rf}$ focusing or by introducing an energy spread within a single bunch as originally proposed [19], or, more recently, by introducing an energy spread between the bunches [20].

Several analyses of what is often referred to as BNS (Balakin-Novokhatsky-Smirnov) damping have been made before [21-30], usually under simplifying assumptions for the current profile, wake function, or time dependence of the parameters. We present here an analysis that provides an exact solution for arbitrary beam current profile, wake function, offset parameters, misalignment of cavities and focusing elements, and time dependence of focusing and BBU coupling strengths. The analytical results are compared to numerical simulations and are found to be in complete agreement.

\section{FORMULATION AND GENERAL SOLUTION}

In a continuum approximation, the transverse motion of a relativistic beam in a misaligned accelerator under the combined influence of focusing and coupling to the dipole modes can be modeled by

$$
\begin{aligned}
& \frac{\partial^{2}}{\partial \sigma^{2}} x(\sigma, \zeta)+\kappa^{2}\left[x(\sigma, \zeta)-d_{f}(\sigma)\right] \\
& \quad=\varepsilon \int_{-\infty}^{\zeta} w\left(\zeta-\zeta_{1}\right) F\left(\zeta_{1}\right)\left[x\left(\sigma, \zeta_{1}\right)-d_{c}(\sigma)\right] d \zeta_{1} .
\end{aligned}
$$

In this expression $\sigma=s / \mathcal{L}$ is the distance from the entrance of the accelerator normalized to the accelerator length $\mathcal{L} ; \kappa$ is the normalized focusing wave number; $\zeta=$ $\omega\left(t-\int d s / c\right)$ is the time made dimensionless by an angular frequency $\omega$ and measured after the arrival of the head of the beam at location $\sigma ; F(\zeta)=I(\zeta) / \bar{I}$, the current form factor, is the instantaneous current divided by the average current; $w(\zeta)$ is the wake function of the dipole modes; $\varepsilon$ is the coupling strength between the beam and the dipole modes; and $d_{f}(\sigma)$ and $d_{c}(\sigma)$ are the lateral displacements of the focusing elements and the 
cavities, respectively, as a function of location along the accelerator.

The dimensionless BBU coupling strength $\varepsilon$ is given by

$$
\varepsilon=\frac{w_{0} \bar{I} e \mathcal{L}^{2}}{\gamma m c^{2} \omega}
$$

where $w_{0}$ is the wake amplitude. With these definitions the wake function $w(\zeta)$ is a dimensionless function of a dimensionless variable and includes only the functional dependence on $\zeta$.

The continuum model assumed in Eq. (1) relies on a number of approximations that are addressed in [17]. Equation (1) also assumes a coasting beam in a uniform accelerator but, as shown in Appendix A of [17], an accelerated beam can, under general assumptions, be reduced to a coasting beam with the introduction of appropriate variable and coordinate transformations.

\section{A. Time-independent parameters}

When $\kappa$ and $\varepsilon$ are constant (independent of $\zeta$ ) Eq. (1) can be solved through the use of the Laplace transform with respect to $\sigma: x^{\dagger}(p, \zeta)=\mathfrak{Q}_{\sigma}[x(\sigma, \zeta)]$. The Laplacetransformed Eq. (1) is

$$
\begin{gathered}
p^{2} x^{\dagger}(p, \zeta)-p x_{0}(\zeta)-x_{0}^{\prime}(\zeta)+\kappa^{2}\left[x^{\dagger}(p, \zeta)-d_{f}^{\dagger}(p)\right] \\
=\varepsilon \int_{-\infty}^{\zeta} w\left(\zeta-\zeta_{1}\right) F\left(\zeta_{1}\right)\left[x^{\dagger}\left(p, \zeta_{1}\right)-d_{c}^{\dagger}(p)\right] d \zeta_{1},
\end{gathered}
$$

and the solutions for $x^{\dagger}(p, \zeta)$ and $x(\sigma, \zeta)$ are [17]

$$
\begin{aligned}
x^{\dagger}(p, \zeta)= & \sum_{n=0}^{\infty} \frac{\varepsilon^{n}}{\left(p^{2}+\kappa^{2}\right)^{n+1}}\left[x_{0} p h_{n}(\zeta)+x_{0}^{\prime} g_{n}(\zeta)\right] \\
& +\kappa^{2} d_{f}^{\dagger}(p) \sum_{n=0}^{\infty} \frac{\varepsilon^{n}}{\left(p^{2}+\kappa^{2}\right)^{n+1}} f_{n}(\zeta) \\
& -d_{c}^{\dagger}(p) \sum_{n=0}^{\infty} \frac{\varepsilon^{n+1}}{\left(p^{2}+\kappa^{2}\right)^{n+1}} f_{n+1}(\zeta)
\end{aligned}
$$

and

$$
\begin{aligned}
x(\sigma, \zeta)= & \sum_{n=0}^{\infty} \varepsilon^{n}\left[x_{0} h_{n}(\zeta) j_{n}(\kappa, \sigma)+x_{0}^{\prime} g_{n}(\zeta) i_{n}(\kappa, \sigma)\right] \\
& +\kappa^{2} \sum_{n=0}^{\infty} \varepsilon^{n} f_{n}(\zeta) i_{n}(\kappa, \sigma) * d_{f}(\sigma) \\
& -\sum_{n=0}^{\infty} \varepsilon^{n+1} f_{n+1}(\zeta) i_{n}(\kappa, \sigma) * d_{c}(\sigma) .
\end{aligned}
$$

The functions $f_{n}(\zeta), g_{n}(\zeta)$, and $h_{n}(\zeta)$ are defined by identical recursion relations

$$
\left\{\begin{array}{l}
f_{n+1}(\zeta) \\
g_{n+1}(\zeta) \\
h_{n+1}(\zeta)
\end{array}\right\}=\int_{-\infty}^{\zeta}\left\{\begin{array}{l}
f_{n}\left(\zeta_{1}\right) \\
g_{n}\left(\zeta_{1}\right) \\
h_{n}\left(\zeta_{1}\right)
\end{array}\right\} F\left(\zeta_{1}\right) w\left(\zeta_{1}-\zeta\right) d \zeta_{1},
$$

with

$$
\begin{aligned}
& f_{0}(\zeta)=1, \\
& g_{0}(\zeta)=x_{0}^{\prime}(\zeta) / x_{0}^{\prime}=\left.\frac{1}{x_{0}^{\prime}} \frac{\partial}{\partial \sigma} x(\sigma, \zeta)\right|_{\sigma=0}, \\
& h_{0}(\zeta)=x_{0}(\zeta) / x_{0}=\frac{1}{x_{0}} x(\sigma=0, \zeta),
\end{aligned}
$$

where $x_{0}(\zeta)$ and $x_{0}^{\prime}(\zeta)$ are the lateral displacement and angular divergence, respectively, of the beam at the entrance of the accelerator. The normalizing constants $x_{0}$ and $x_{0}^{\prime}$ are introduced to make the functions $h_{0}(\zeta)$ and $g_{0}(\zeta)$ dimensionless.

The functions $i_{n}(\kappa, \sigma)$ and $j_{n}(\kappa, \sigma)$ are defined in terms of Bessel functions of order integer plus one half

$$
\begin{aligned}
i_{n}(\kappa, \sigma) & =\mathfrak{Q}_{\sigma}^{-1}\left[\frac{1}{\left(p^{2}+\kappa^{2}\right)^{n+1}}\right] \\
& =\frac{1}{n !}\left(\frac{\sigma}{2 \kappa}\right)^{n} \frac{1}{\kappa} \sqrt{\frac{\pi \kappa \sigma}{2}} J_{n+(1 / 2)}(\kappa \sigma), \\
j_{n}(\kappa, \sigma) & =\mathfrak{Q}_{\sigma}^{-1}\left[\frac{p}{\left(p^{2}+\kappa^{2}\right)^{n+1}}\right] \\
& =\frac{1}{n !}\left(\frac{\sigma}{2 \kappa}\right)^{n} \sqrt{\frac{\pi \kappa \sigma}{2}} J_{n-(1 / 2)}(\kappa \sigma),
\end{aligned}
$$

and

$$
i_{n}(\kappa, \sigma) * d(\sigma)=\int_{0}^{\sigma} i_{n}(\kappa, u) d(\sigma-u) d u
$$

is the convolution of $i_{n}(\kappa, \sigma)$ and $d(\sigma)$.

Applications of these results were presented in $[17,18]$.

\section{B. Time-dependent parameters}

When the strengths of the focusing and of the coupling between the beam and the dipole modes are time dependent $[\varepsilon(\zeta)$ and $\kappa(\zeta)]$ the beam displacement governed by Eq. (1) is not given by Eq. (5) any more and the procedure for solving Eqs. (1) and (3) needs to be modified since, as shown in Appendix B of [17], to obtain Eqs. (4)-(6) $\varepsilon$ and $\kappa$ were assumed to be independent of $\zeta$ in order to be taken out of the integrals. This can be done by splitting the focusing strength $\kappa(\zeta)$ in two parts, one constant and one time dependent, such that

$$
\kappa^{2}(\zeta)=\kappa_{0}^{2}[1+\Delta \kappa(\zeta)]=\kappa_{0}^{2}+\kappa_{1}^{2}(\zeta) .
$$

As shown in the Appendix, the displacement $x(\sigma, \zeta)$ and its Laplace transform $x^{\dagger}(p, \zeta)$ are then given by 


$$
\begin{aligned}
x^{\dagger}(p, \zeta)= & \sum_{n=0}^{\infty} \frac{1}{\left(p^{2}+\kappa_{0}^{2}\right)^{n+1}}\left[p x_{0} h_{n}^{\star}(\zeta)+x_{0}^{\prime} g_{n}^{\star}(\zeta)\right]+\kappa_{0}^{2} d_{f}^{\dagger}(p) \sum_{n=0}^{\infty} \frac{1}{\left(p^{2}+\kappa_{0}^{2}\right)^{n+1}} k_{n}^{\star}(\zeta) \\
& -d_{c}^{\dagger}(p) \sum_{n=0} \frac{1}{\left(p^{2}+\kappa_{0}^{2}\right)^{n+1}}\left\{f_{n+1}^{\star}(\zeta)+\kappa_{0}^{2}\left[k_{n}^{\star}(\zeta)-f_{n}^{\star}(\zeta)\right]\right\}, \\
x(\sigma, \zeta)= & \sum_{n=0}^{\infty}\left[x_{0} j_{n}\left(\kappa_{0}, \sigma\right) h_{n}^{\star}(\zeta)+x_{0}^{\prime} i_{n}\left(\kappa_{0}, \sigma\right) g_{n}^{\star}(\zeta)\right]+\kappa_{0}^{2} \sum_{n=0}^{\infty} k_{n}^{\star}(\zeta) i_{n}\left(\kappa_{0}, \sigma\right) * d_{f}(\sigma) \\
& -\sum_{n=0}^{\infty}\left\{f_{n+1}^{\star}(\zeta)+\kappa_{0}^{2}\left[k_{n}^{\star}(\zeta)-f_{n}^{\star}(\zeta)\right]\right\} i_{n}\left(\kappa_{0}, \sigma\right) * d_{c}(\sigma),
\end{aligned}
$$

where the functions $f_{n}^{\star}(\zeta), g_{n}^{\star}(\zeta), h_{n}^{\star}(\zeta)$, and $k_{n}^{\star}(\zeta)$ satisfy identical recursion relations

$$
\begin{aligned}
\left\{\begin{array}{l}
f_{n+1}^{\star}(\zeta) \\
g_{n+1}^{\star}(\zeta) \\
h_{n+1}^{\star}(\zeta) \\
k_{n+1}^{\star}(\zeta)
\end{array}\right\}= & \varepsilon(\zeta) \int_{-\infty}^{\zeta}\left\{\begin{array}{l}
f_{n}^{\star}\left(\zeta_{1}\right) \\
g_{n}^{\star}\left(\zeta_{1}\right) \\
h_{n}^{\star}\left(\zeta_{1}\right) \\
k_{n}^{\star}\left(\zeta_{1}\right)
\end{array}\right\} w\left(\zeta-\zeta_{1}\right) F\left(\zeta_{1}\right) d \zeta_{1} \\
& -\kappa_{1}^{2}(\zeta)\left\{\begin{array}{l}
f_{n}^{\star}(\zeta) \\
g_{n}^{\star}(\zeta) \\
h_{n}^{\star}(\zeta) \\
k_{n}^{\star}(\zeta)
\end{array}\right\}
\end{aligned}
$$

and

$$
\begin{aligned}
f_{0}^{\star}(\zeta) & =1, \\
g_{0}^{\star}(\zeta) & =x_{0}^{\prime}(\zeta) / x_{0}^{\prime}, \\
h_{0}^{\star}(\zeta) & =x_{0}(\zeta) / x_{0}, \\
k_{0}^{\star}(\zeta) & =\frac{\kappa^{2}(\zeta)}{\kappa_{0}^{2}}=1+\frac{\kappa_{1}^{2}(\zeta)}{\kappa_{0}^{2}}=1+\Delta \kappa(\zeta), \\
i_{n}\left(\kappa_{0}, \sigma\right) & =\frac{1}{n !}\left(\frac{\sigma}{2 \kappa_{0}}\right)^{n} \frac{1}{\kappa_{0}} \sqrt{\frac{\pi \kappa_{0} \sigma}{2}} J_{n+(1 / 2)}\left(\kappa_{0} \sigma\right), \\
j_{n}\left(\kappa_{0}, \sigma\right) & =\frac{1}{n !}\left(\frac{\sigma}{2 \kappa_{0}}\right)^{n} \sqrt{\frac{\pi \kappa_{0} \sigma}{2}} J_{n-(1 / 2)}\left(\kappa_{0} \sigma\right) .
\end{aligned}
$$

Equation (12) gives the transverse displacement, at location $\sigma$ and time $\zeta$, of a beam of arbitrary current $F(\zeta)$, entering the accelerator with lateral offset $x_{0}(\zeta)$ and angular divergence $x_{0}^{\prime}(\zeta)$, experiencing transverse forces due to coupling $\varepsilon(\zeta)$ to cavity dipole modes of wakefield $w(\zeta)$ and to focusing $\kappa(\zeta)$, and with displacement along the accelerator $d_{c}(\sigma)$ of the cavities and $d_{f}(\sigma)$ of the focusing elements.

There is some arbitrariness in the way the focusing strength $\kappa(\zeta)$ is split in two parts according to Eq. (10). For example, it could be assumed that $\kappa(\zeta)$ has no constant term $\left(\kappa_{0}=0\right)$ and only a time-dependent term. In this case $x^{\dagger}(p, \zeta)$ and $x(\sigma, \zeta)$ would be given by

$$
\begin{aligned}
x^{\dagger}(p, \zeta)= & \sum_{n=0}^{\infty}\left[x_{0} \frac{h_{n}^{\circ}(\zeta)}{p^{2 n+1}}+x_{0}^{\prime} \frac{g_{n}^{\circ}(\zeta)}{p^{2 n+2}}\right]+\bar{\kappa}^{2} d_{f}^{\dagger} \sum_{n=0}^{\infty} \frac{k_{n}^{\circ}(\zeta)}{p^{2 n+2}} \\
& -d_{c}^{\dagger}(p) \sum_{n=0}^{\infty} \frac{\left[f_{n+1}^{\circ}(\zeta)+\bar{\kappa}^{2} k_{n}^{\circ}(\zeta)\right]}{p^{2 n+2}} \\
x(\sigma, \zeta)= & \sum_{n=0}^{\infty}\left[x_{0} \frac{\sigma^{2 n}}{2 n !} h_{n}^{\circ}(\zeta)+x_{0}^{\prime} \frac{\sigma^{2 n+1}}{(2 n+1) !} g_{n}^{\circ}(\zeta)\right] \\
& +\bar{\kappa}^{2} \sum_{n=0}^{\infty} k_{n}^{\circ}(\zeta) \frac{\sigma^{2 n+1} * d_{f}(\sigma)}{(2 n+1) !} \\
& -\sum_{n=0}^{\infty}\left[f_{n+1}^{\circ}(\zeta)+\bar{\kappa}^{2} k_{n}^{\circ}(\zeta)\right] \frac{\sigma^{2 n+1} * d_{c}(\sigma)}{(2 n+1) !}
\end{aligned}
$$

where the functions $f_{n}^{\circ}(\zeta), g_{n}^{\circ}(\zeta), h_{n}^{\circ}(\zeta)$, and $k_{n}^{\circ}(\zeta)$ satisfy identical recursion relations

$$
\begin{gathered}
\left\{\begin{array}{l}
f_{n+1}^{\circ}(\zeta) \\
g_{n+1}^{\circ}(\zeta) \\
h_{n+1}^{\circ}(\zeta) \\
k_{n+1}^{\circ}(\zeta)
\end{array}\right\}= \\
=\varepsilon(\zeta) \int_{-\infty}^{\zeta}\left\{\begin{array}{l}
f_{n}^{\circ}\left(\zeta_{1}\right) \\
g_{n}^{\circ}\left(\zeta_{1}\right) \\
h_{n}^{\circ}\left(\zeta_{1}\right) \\
k_{n}^{\circ}\left(\zeta_{1}\right)
\end{array}\right\} w\left(\zeta-\zeta_{1}\right) F\left(\zeta_{1}\right) d \zeta_{1} \\
-\kappa^{2}(\zeta)\left\{\begin{array}{l}
f_{n}^{\circ}(\zeta) \\
g_{n}^{\circ}(\zeta) \\
h_{n}^{\circ}(\zeta) \\
k_{n}^{\circ}(\zeta)
\end{array}\right\}
\end{gathered}
$$

and

$$
\begin{aligned}
& f_{0}^{\circ}(\zeta)=1, \\
& g_{0}^{\circ}(\zeta)=x_{0}^{\prime}(\zeta) / x_{0}^{\prime}, \\
& h_{0}^{\circ}(\zeta)=x_{0}(\zeta) / x_{0}, \\
& k_{0}^{\circ}(\zeta)=\frac{\kappa^{2}(\zeta)}{\bar{\kappa}^{2}} .
\end{aligned}
$$

The arbitrary constant $\bar{\kappa}$ is introduced only to make the function $k_{0}^{\circ}(\zeta)$ dimensionless - similar to $f_{0}^{\circ}(\zeta), g_{0}^{\circ}(\zeta)$, and $h_{0}^{\circ}(\zeta)$ - and the final result given by Eq. (17) is independent of its choice. 
While the expressions (11)-(15) and (16)-(19) for $x^{\dagger}(p, \zeta)$ and $x(\sigma, \zeta)$ look very different, they are mathematically equivalent and represent the same solutions of Eqs. (1) and (3). They differ significantly, however, in their rate of convergence, with expressions (16)-(19) converging very slowly. For expressions (11)-(15) to be of practical use, the separation of $\kappa^{2}(\zeta)$ in two parts, as given by Eq. (10), needs to be done in such a way that the timedependent part $\Delta \kappa(\zeta)$ is kept as small as possible. Since, in practical applications, the focusing strength will change only by a small amount, natural choices for $\kappa_{0}^{2}$ would be the value of $\kappa^{2}(\zeta)$ either at the beginning, the end, or its average value while the beam is in the accelerator.

Equations (11)-(15) are very general. For the remainder of this paper, in order to simplify the equations, we will assume that the accelerator is perfectly aligned $\left[d_{f}(\sigma)=\right.$ $d_{c}(\sigma)=0$ ], that the injection offsets are time independent $\left[x_{0}^{\prime}(\zeta)=x_{0}^{\prime}, x_{0}(\zeta)=x_{0}\right]$, and that the beam was turned on at $\zeta=0[F(\zeta<0)=0]$. Extension of the following results to time-dependent injection offsets and misaligned accelerators is straightforward by applying the results of $[17,18]$. by

With these assumptions $x^{\dagger}(p, \zeta)$ and $x(\sigma, \zeta)$ are given

$$
\begin{aligned}
& x^{\dagger}(p, \zeta)=\sum_{n=0}^{\infty} \frac{x_{0}^{\prime}+p x_{0}}{\left(p^{2}+\kappa_{0}^{2}\right)^{n+1}} f_{n}^{\star}(\zeta), \\
x(\sigma, \zeta)= & \sum_{n=0}^{\infty}\left[x_{0} j_{n}\left(\kappa_{0}, \sigma\right)+x_{0}^{\prime} i_{n}\left(\kappa_{0}, \sigma\right)\right] f_{n}^{\star}(\zeta), \\
f_{0}^{\star}(\zeta)= & 1, \\
f_{n+1}^{\star}(\zeta)= & \varepsilon(\zeta) \int_{0}^{\zeta} w\left(\zeta-\zeta_{1}\right) F\left(\zeta_{1}\right) f_{n}^{\star}\left(\zeta_{1}\right) d \zeta_{1} \\
& -\kappa_{1}^{2}(\zeta) f_{n}^{\star}(\zeta) .
\end{aligned}
$$

From Eqs. (22) we see that

$$
f_{1}^{\star}(\zeta)=\varepsilon(\zeta) \int_{0}^{\zeta} w\left(\zeta_{1}-\zeta\right) F\left(\zeta_{1}\right) d \zeta_{1}-\kappa_{1}^{2}(\zeta) .
$$

Thus, by choosing

$$
\kappa_{1}^{2}(\zeta)=\varepsilon(\zeta) \int_{0}^{\zeta} w\left(\zeta-\zeta_{1}\right) F\left(\zeta_{1}\right) d \zeta_{1}
$$

we have

$$
f_{n>0}^{\star}(\zeta)=0
$$

and

$$
\begin{aligned}
x(\sigma, \zeta) & =x_{0} j_{0}\left(\kappa_{0} \sigma\right)+x_{0}^{\prime} i_{0}\left(\kappa_{0} \sigma\right) \\
& =x_{0} \cos \kappa_{0} \sigma+\frac{x_{0}^{\prime}}{\kappa_{0}} \sin \kappa_{0} \sigma .
\end{aligned}
$$

As a result the beam progresses in the accelerator as though it were only experiencing a constant focusing force without interaction with the deflecting mode. Equation (24) is the general condition for complete sup- pression of BBU by imposing a time dependence on the focusing or the coupling strength. This condition is often referred to as autophasing [23].

It should be noted that the autophasing condition Eq. (24) that eliminated the BBU instability was obtained in the case of a perfectly aligned accelerator with timeindependent injection offsets. As can be seen from Eq. (12), the autophasing condition would still be effective with time-independent offsets and identical displacements of cavities and focusing elements $\left[d_{c}(\sigma)=d_{f}(\sigma)\right]$ but would not be effective if the injection offsets were time dependent, or if the misalignments were different for the cavities and the focusing elements.

\section{SINGLE VERY SHORT BUNCH}

The results of the previous section will be first applied to the case of a single, very short bunch. In this case the wake function is assumed to be linear $[w(\zeta)=\zeta]$ and the current density along the bunch is assumed to be constant $[F(\zeta)=1]$. We will further assume that the BBU coupling strength is constant.

If the time dependence of the focusing is assumed to be quadratic

$$
\kappa^{2}(\zeta)=\kappa_{0}^{2}\left[1+\eta \zeta^{2}\right]
$$

then the functions $f_{n}^{*}(\zeta)$ can be easily calculated:

$$
\begin{aligned}
f_{0}^{\star} & =1, \\
f_{n}^{\star}(\zeta) & =\frac{\zeta^{2 n}}{(2 n) !} \prod_{k=1}^{n}\left[\varepsilon-(2 k-1)(2 k) \kappa_{0}^{2} \eta\right] .
\end{aligned}
$$

This, together with Eq. (21), defines completely the displacement $x(\sigma, \zeta)$. If the focusing modulation parameter $\eta$ is chosen such that $\eta=\varepsilon /\left(2 \kappa_{0}^{2}\right)$, then $f_{n>0}^{\star}(\zeta)=0$ and the coupling between the beam and the dipole mode is completely suppressed.

In the case of a linear time dependence of the focusing,

$$
\kappa^{2}(\zeta)=\kappa_{0}^{2}[1+\eta \zeta]
$$

the functions $f_{n}^{\star}(\zeta)$ can be obtained through the recursion relations

$$
\begin{aligned}
& f_{n}^{\star}(\zeta)=\sum_{k=n}^{2 n} a_{n, k} \zeta^{k}, \quad a_{0,0}=1, \\
& a_{n, k}=\frac{\varepsilon a_{n-1, k-2}}{k(k-1)}-\kappa_{0}^{2} \eta a_{n-1, k-1} .
\end{aligned}
$$

In particular,

$$
\begin{aligned}
& f_{0}^{\star}(\zeta)=1, \\
& f_{1}^{\star}(\zeta)=\frac{\varepsilon}{2} \zeta^{2}-\kappa_{0}^{2} \eta \zeta, \\
& f_{2}^{\star}(\zeta)=\frac{\varepsilon^{2}}{24} \zeta^{4}-\frac{2}{3} \varepsilon \kappa_{0}^{2} \eta \zeta^{3}+\kappa_{0}^{4} \eta^{2} \zeta^{2} .
\end{aligned}
$$

These results are illustrated in Fig. 1, which shows the shape of a bunch of length $\zeta_{b}$ at the exit $(\sigma=1)$ of an 

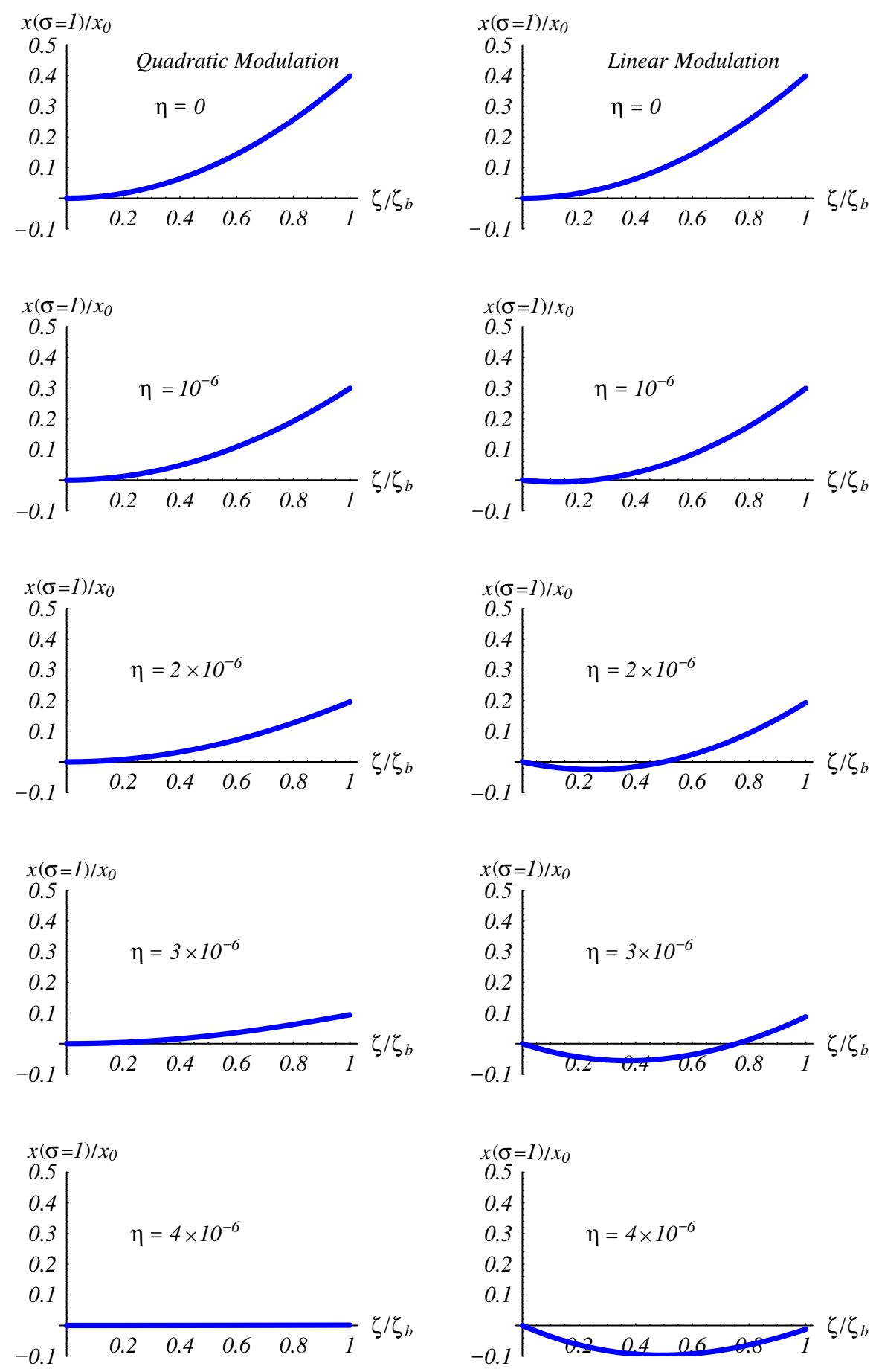

FIG. 1. (Color) Bunch shape at the exit of the accelerator as a function of focusing modulation for a quadratic (left) or linear (right) time dependence of the modulation. The parameter $\eta$ is the amount of focusing modulation defined by Eqs. (27) and (29). See text for the other parameters.

accelerator for quadratic (left) and linear (right) time dependence of the focusing modulation. The parameter $\eta$ represents the rate of modulation of the focusing as defined by Eqs. (27) and (29). The focusing strength is set at $\kappa_{0}=$ $100.5 \pi$; this implies that, since $\sigma=1$, the lateral displacement is due entirely to the coupling with the deflecting mode and to the focusing modulation, and not to the betatron motion without focusing modulation. The strength of the coupling to the dipole mode is assumed to be $\varepsilon=$ 0.8 and the length of the bunch is assumed to be $\zeta_{b}=1$. Figure 1 illustrates how the right amount of quadratic modulation can cancel the effect of the coupling to the dipole modes, while a linear modulation can reduce it but not eliminate it. 
Such modulations could be provided by rf focusing; a linear modulation would be obtained by operating the rf focusing elements sufficiently far off crest with respect to the beam, while a quadratic modulation would be obtained by operating the focusing elements on crest. A similar effect could be obtained by introducing linear or quadratic energy modulations along the bunch [31] .

\section{FINITE TRAIN OF POINTLIKE BUNCHES}

The results of Sec. II will be applied here to a finite train of $N$ identical pointlike bunches separated, in the laboratory frame, by $\tau$, so that bunch $M$ is defined by $\zeta=M \omega \tau$. The displacement of bunch $M$ is then given by

$$
\begin{gathered}
x_{M}(\sigma)=\sum_{n=0}^{\infty} f_{n}^{\star}(M \omega \tau)\left[x_{0} j_{0}\left(\kappa_{0}, \sigma\right)+x_{0}^{\prime} i_{0}\left(\kappa_{0}, \sigma\right)\right], \\
f_{n+1}^{\star}(M \omega \tau)=\omega \tau \varepsilon(M \omega \tau) \sum_{k=0}^{M} f_{n}^{\star}(k \omega \tau) w[(M-k) \omega \tau] \\
-\kappa_{0}^{2} \Delta \kappa(M \omega \tau) f_{n}^{\star}(M \omega \tau) .
\end{gathered}
$$

As an example, the analytical results expressed by Eqs. (32) and (33) will be applied to a beam representative
TABLE I. Nominal top-level linear-collider design parameters $[17,18,28,29]$.

\begin{tabular}{lc}
\hline \hline \multicolumn{1}{c}{ Parameter } & Value \\
\hline Total initial energy $\gamma(0) m c^{2}$ & $10 \mathrm{GeV}$ \\
Total final energy $\gamma(1) m c^{2}$ & $1 \mathrm{TeV}$ \\
Linac length $\mathcal{L}$ & $10 \mathrm{~km}$ \\
Number of betatron periods & 100 \\
Bunch charge & $1 \mathrm{nC}$ \\
Number of bunches in train $N$ & 90 \\
Bunch spacing $\tau$ & $2.8 \mathrm{~ns}$ \\
Deflecting-wake frequency $\omega / 2 \pi$ & $14.95 \mathrm{GHz}$ \\
Deflecting-wake amplitude $w_{0}$ & $10^{15} \mathrm{~V} \mathrm{C}^{-1} \mathrm{~m}^{-1}$ \\
\hline \hline
\end{tabular}

of a linear collider. For comparison we will use the same parameters as those used in [17,18,28,29] and listed in Table I. Since this is an accelerated beam, the transformations described in Appendix A of [17] will be used. Converting the parameters in Table I to those used in this paper we have $\varepsilon(\sigma=0)=\frac{w_{0} q e \mathcal{L}^{2}}{\gamma(0) m c^{2} \omega \tau}=38.02, \kappa(\sigma=$ $0)=1100 \pi, \varsigma(\sigma=1)=2 / 11$, and $\omega \tau=263.014$. The wake function is assumed to be of the form $w(\zeta)=u(\zeta) \times$ $\sin \zeta \exp [-\zeta /(2 Q)]$.
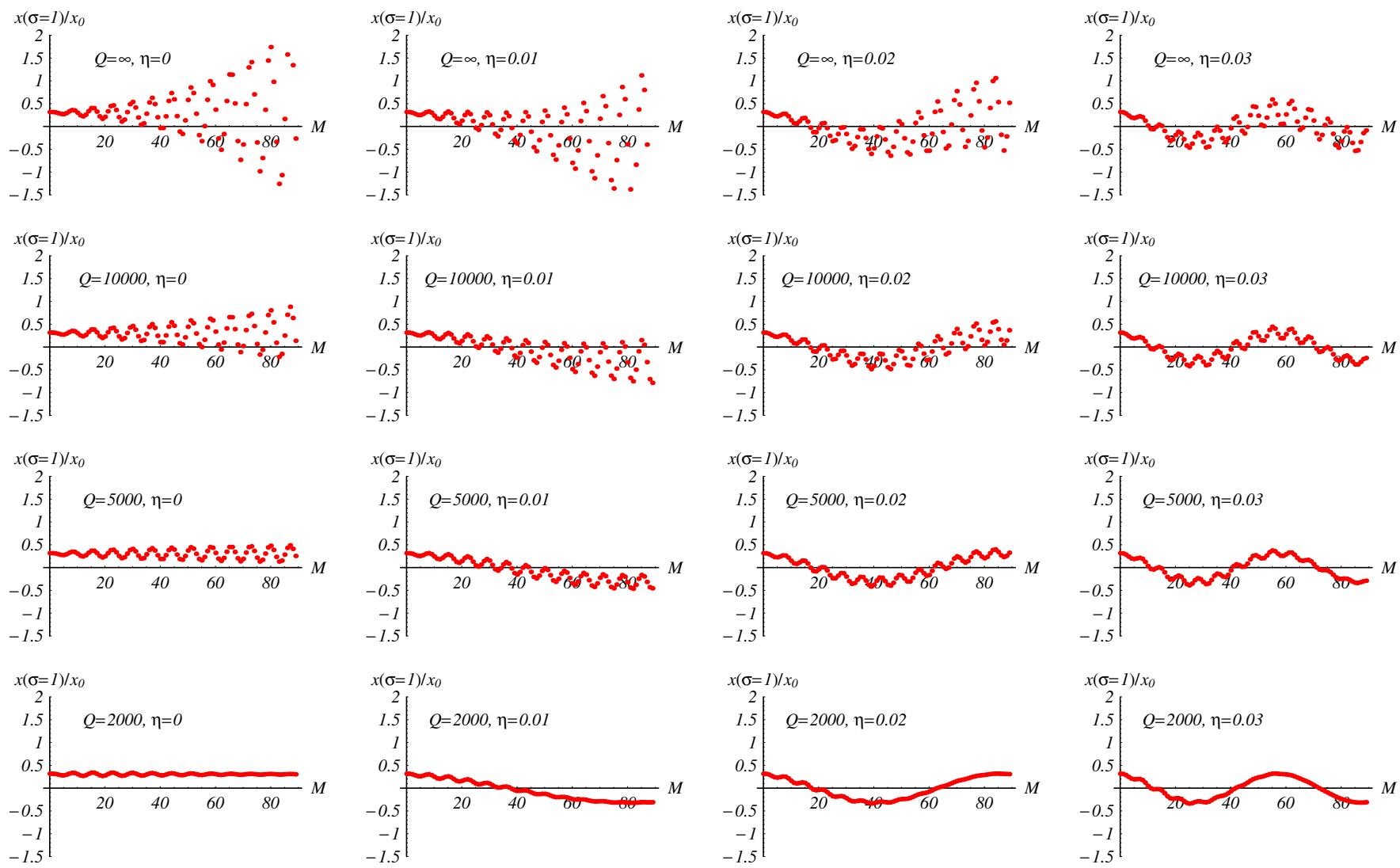

FIG. 2. (Color) Normalized lateral displacement of a finite train of pointlike bunches at the exit of a representative linear collider for different values of the $Q$ of the deflecting mode and of the modulation of the focusing strength. See Table I for the choice of parameters. 
This example illustrates the relative effects of a finite $Q$ of the deflecting mode and of a time-dependent focusing strength of the form

$$
\kappa^{2}(M \omega \tau)=\kappa_{0}^{2}\left[1+\eta \frac{M}{N-1}\right]
$$

where $M$ (from $M=0$ to $M=N-1$ ) is the bunch number and $N$ is the total number of bunches.

The transverse displacement at location $\sigma$ for bunch $M$ is given by Eq. (32), where the functions $f_{n}^{\star}(M \omega \tau)$ are calculated using the recursion relation of Eq. (33). The transverse displacements of the bunches at the exit of the accelerator for the beam and accelerator parameters of Table I are shown in Fig. 2. The rows are (top to bottom) for a $Q$ of the deflecting mode of $\infty, 10000,5000$, and 2000. The columns are (left to right) for modulation parameter $\eta$ of $0,0.01,0.02$, and 0.03 .

The top right-hand plot in Fig. $2(Q=\infty, \eta=0.03)$, which was obtained analytically, is identical in all details to Fig. 4 of [29], which was obtained numerically by tracking successive bunches as they progress along the same accelerator.

While this example assumes a linear time dependence of the focusing strength and a simple wake function, other more complicated profiles and wake functions can be as easily accommodated by the recursion relations [Eq. (33)].

\section{SUMMARY}

This paper presents a formalism to address analytically cumulative beam breakup in linear accelerators with timedependent parameters. Such time dependence, which could result from an energy chirp imposed on the beam or from the use of rf focusing, can be effective in reducing the coupling between the beam and the dipole modes.

This formalism allows, in principle, direct calculation, for any time and any position in an accelerator, of the transverse displacement of a beam of arbitrary current profile, entering the accelerator with arbitrary timedependent lateral displacement and angular divergence, and with random displacements of the cavities and focusing elements.

When applied to a colliderlike accelerator, the analytical results presented here reproduce exactly the numerical simulations that were done previously.

\section{ACKNOWLEDGMENTS}

This work was supported by the U.S. Department of Energy under Contracts No. DE-AC05-84-ER40150 and No. DE-AC05-00-OR22725.

\section{APPENDIX: GENERAL SOLUTION OF THE EQUATION OF MOTION}

The equation of motion for the transverse displacement is

$$
\frac{\partial^{2}}{\partial \sigma^{2}} x(\sigma, \zeta)+\kappa^{2}(\zeta)\left[x(\sigma, \zeta)-d_{f}(\sigma)\right]=\varepsilon(\zeta) \int_{-\infty}^{\zeta} w\left(\zeta-\zeta_{1}\right) F\left(\zeta_{1}\right)\left[x\left(\sigma, \zeta_{1}\right)-d_{c}(\sigma)\right] d \zeta_{1} .
$$

We separate the focusing in two parts, one constant and one time dependent, such that

$$
\kappa^{2}(\zeta)=\kappa_{0}^{2}[1+\Delta \kappa(\zeta)]=\kappa_{0}^{2}+\kappa_{1}^{2}(\zeta),
$$

and we apply the Laplace transform with respect to $\sigma$ to obtain

$$
\begin{aligned}
& p^{2} x^{\dagger}(p, \zeta)-p x_{0}(\zeta)-x_{0}^{\prime}(\zeta)+\kappa_{0}^{2}\left[x^{\dagger}(p, \zeta)-d_{f}^{\dagger}(p)\right]+\kappa_{1}^{2}(\zeta)\left[x^{\dagger}(p, \zeta)-d_{f}^{\dagger}(p)\right] \\
& \quad=\varepsilon(\zeta) \int_{-\infty}^{\zeta} w\left(\zeta-\zeta_{1}\right) F\left(\zeta_{1}\right)\left[x^{\dagger}\left(p, \zeta_{1}\right)-d_{c}^{\dagger}(p)\right] d \zeta_{1} .
\end{aligned}
$$

By analogy with the time-independent case [where $\kappa_{1}^{2}(\zeta)=0$ ], we assume that $x^{\dagger}(p, \zeta)$ is of the form

$$
x^{\dagger}(p, \zeta)=\sum_{n=0}^{\infty} \frac{1}{\left(p^{2}+\kappa_{0}^{2}\right)^{n+1}}\left[p x_{0} h_{n}^{\star}(\zeta)+x_{0}^{\prime} g_{n}^{\star}(\zeta)\right]+\kappa_{0}^{2} d_{f}^{\dagger}(p) \sum_{n=0}^{\infty} \frac{1}{\left(p^{2}+\kappa_{0}^{2}\right)^{n+1}} k_{n}^{\star}(\zeta)-d_{c}^{\dagger}(p) \sum_{n=0} \frac{1}{\left(p^{2}+\kappa_{0}^{2}\right)^{n+1}} l_{n+1}^{\star}(\zeta)
$$

where the functions $g_{n}^{\star}(\zeta), h_{n}^{\star}(\zeta), k_{n}^{\star}(\zeta)$, and $l_{n}^{\star}(\zeta)$ are to be determined.

We see from Eq. (A4) that the transverse displacement is a linear combination of the displacements caused by the injection offsets and the misalignments.

To determine $g_{n}^{\star}(\zeta)$, we assume that $x_{0}(\zeta)=0$, and $d_{f}(\sigma)=d_{c}(\sigma)=0$, so that $x^{\dagger}(p, \zeta)$ reduces to

$$
x^{\dagger}(p, \zeta)=x_{0}^{\prime} \sum_{n=0}^{\infty} \frac{1}{\left(p^{2}+\kappa_{0}^{2}\right)^{n+1}} g_{n}^{\star}(\zeta)
$$

and must satisfy 


$$
\left[p^{2}+\kappa_{0}^{2}\right] x^{\dagger}(p, \zeta)+\kappa_{1}^{2}(\zeta) x^{\dagger}(p, \zeta)-x_{0}^{\prime}(\zeta)=\varepsilon(\zeta) \int_{-\infty}^{\zeta} w\left(\zeta-\zeta_{1}\right) F\left(\zeta_{1}\right) x^{\dagger}\left(p, \zeta_{1}\right) d \zeta_{1}
$$

This implies that

$$
\sum_{n=0}^{\infty} \frac{1}{\left(p^{2}+\kappa_{0}^{2}\right)^{n+1}}\left[g_{n+1}^{\star}(\zeta)+\kappa_{1}^{2}(\zeta) g_{n}^{\star}(\zeta)-\varepsilon(\zeta) \int_{-\infty}^{\zeta} w\left(\zeta-\zeta_{1}\right) F\left(\zeta_{1}\right) g_{n}^{\star}\left(\zeta_{1}\right) d \zeta_{1}\right]+g_{0}^{\star}(\zeta)-\frac{x_{0}^{\prime}(\zeta)}{x_{0}^{\prime}}=0
$$

which leads to

$$
\begin{aligned}
g_{0}^{\star}(\zeta) & =\frac{x_{0}^{\prime}(\zeta)}{x_{0}^{\prime}} \\
g_{n+1}^{\star}(\zeta) & =\varepsilon(\zeta) \int_{-\infty}^{\zeta} w\left(\zeta-\zeta_{1}\right) F\left(\zeta_{1}\right) g_{n}^{\star}\left(\zeta_{1}\right) d \zeta_{1}-\kappa_{1}^{2}(\zeta) g_{n}^{\star}(\zeta) .
\end{aligned}
$$

The functions $h_{n}^{\star}(\zeta)$ are determined in a similar fashion by assuming $x_{0}^{\prime}(\zeta)=0$, and $d_{f}(\sigma)=d_{c}(\sigma)=0$. They satisfy the same recursion relation as $g_{n}^{\star}(\zeta)$ but with $h_{0}^{\star}(\zeta)=x_{0}(\zeta) / x_{0}$.

To determine $k_{n}^{\star}(\zeta)$ we assume that $x_{0}(\zeta)=x_{0}^{\prime}(\zeta)=0$ and $d_{c}(\sigma)=0$, so that $x^{\dagger}(p, \sigma)$ reduces to

$$
x^{\dagger}(p, \sigma)=\kappa_{0}^{2} d_{f}^{\dagger}(p) \sum_{n=0}^{\infty} \frac{1}{\left(p^{2}+\kappa_{0}^{2}\right)^{n+1}} k_{n}^{\star}(\zeta)
$$

and must satisfy

$$
\left[p^{2}+\kappa_{0}^{2}\right] x^{\dagger}(p, \zeta)+\kappa_{1}^{2}(\zeta) x^{\dagger}(p, \zeta)-\kappa^{2}(\zeta) d_{f}^{\dagger}(p)=\varepsilon(\zeta) \int_{-\infty}^{\zeta} w\left(\zeta-\zeta_{1}\right) F\left(\zeta_{1}\right) x^{\dagger}\left(p, \zeta_{1}\right) d \zeta_{1}
$$

This implies that

$$
\sum_{n=0}^{\infty} \frac{1}{\left(p^{2}+\kappa_{0}^{2}\right)^{n+1}}\left[k_{n+1}^{\star}(\zeta)+\kappa_{1}^{2}(\zeta) k_{n}^{\star}(\zeta)-\varepsilon(\zeta) \int_{-\infty}^{\zeta} w\left(\zeta-\zeta_{1}\right) F\left(\zeta_{1}\right) k_{n}^{\star}\left(\zeta_{1}\right) d \zeta_{1}\right]+k_{0}^{\star}(\zeta)-\frac{\kappa^{2}(\zeta)}{\kappa_{0}^{2}}=0,
$$

which leads to

$$
\begin{aligned}
k_{0}^{\star}(\zeta) & =\frac{\kappa^{2}(\zeta)}{\kappa_{0}^{2}}=1+\frac{\kappa_{1}^{2}(\zeta)}{\kappa_{0}^{2}}=1+\Delta \kappa(\zeta), \\
k_{n+1}^{\star}(\zeta) & =\varepsilon(\zeta) \int_{-\infty}^{\zeta} w\left(\zeta-\zeta_{1}\right) F\left(\zeta_{1}\right) k_{n}^{\star}\left(\zeta_{1}\right) d \zeta_{1}-\kappa_{1}^{2}(\zeta) k_{n}^{\star}(\zeta) .
\end{aligned}
$$

To determine $l_{n}^{\star}(\zeta)$ we assume that $x_{0}(\zeta)=x_{0}^{\prime}(\zeta)=0$ and $d_{f}(\sigma)=0$, so that $x^{\dagger}(p, \sigma)$ reduces to

$$
x^{\dagger}(p, \sigma)=-d_{c}^{\dagger}(p) \sum_{n=0}^{\infty} \frac{1}{\left(p^{2}+\kappa_{0}^{2}\right)^{n+1}} l_{n+1}^{\star}(\zeta)
$$

and must satisfy

$$
\left[p^{2}+\kappa_{0}^{2}\right] x^{\dagger}(p, \zeta)+\kappa_{1}^{2}(\zeta) x^{\dagger}(p, \zeta)=\varepsilon(\zeta) \int_{-\infty}^{\zeta} w\left(\zeta-\zeta_{1}\right) F\left(\zeta_{1}\right)\left[x^{\dagger}\left(p, \zeta_{1}\right)-d_{c}^{\dagger}(p)\right] d \zeta_{1} .
$$

This implies that

$$
\sum_{n=0}^{\infty} \frac{1}{\left(p^{2}+\kappa_{0}^{2}\right)^{n+1}}\left[l_{n+2}^{\star}(\zeta)+\kappa_{1}^{2}(\zeta) l_{n+1}^{\star}(\zeta)-\varepsilon(\zeta) \int_{-\infty}^{\zeta} w\left(\zeta-\zeta_{1}\right) F\left(\zeta_{1}\right) l_{n+1}^{\star}\left(\zeta_{1}\right) d \zeta_{1}\right]+l_{1}^{\star}(\zeta)-\varepsilon(\zeta) \int_{-\infty}^{\zeta} w\left(\zeta-\zeta_{1}\right) F\left(\zeta_{1}\right) d \zeta_{1}=0
$$

which leads to 


$$
\begin{aligned}
l_{1}^{\star}(\zeta) & =\varepsilon(\zeta) \int_{-\infty}^{\zeta} w\left(\zeta-\zeta_{1}\right) F\left(\zeta_{1}\right) d \zeta_{1}, \\
l_{n+1}^{\star}(\zeta) & =\varepsilon(\zeta) \int_{-\infty}^{\zeta} w\left(\zeta-\zeta_{1}\right) F\left(\zeta_{1}\right) l_{n}^{\star}\left(\zeta_{1}\right) d \zeta_{1}-\kappa_{1}^{2}(\zeta) l_{n}^{\star}(\zeta)
\end{aligned}
$$

If we define the functions $f_{n}^{\star}(\zeta)$ by

$$
\begin{aligned}
f_{0}^{\star}(\zeta) & =1 \\
f_{n+1}^{\star}(\zeta) & =\varepsilon(\zeta) \int_{-\infty}^{\zeta} w\left(\zeta-\zeta_{1}\right) F\left(\zeta_{1}\right) f_{n}^{\star}\left(\zeta_{1}\right) d \zeta_{1}-\kappa_{1}^{2}(\zeta) f_{n}^{\star}(\zeta),
\end{aligned}
$$

then

$$
l_{1}^{\star}(\zeta)=f_{1}^{\star}(\zeta)+\kappa_{1}^{2}(\zeta)=f_{1}^{\star}(\zeta)+\kappa_{0}^{2}\left[k_{0}^{\star}(\zeta)-f_{0}^{\star}(\zeta)\right] .
$$

Since the functions $f_{n}^{\star}(\zeta), k_{n}^{\star}(\zeta)$, and $l_{n}^{\star}(\zeta)$ satisfy the same recursion relation, we obtain

$$
l_{n+1}^{\star}(\zeta)=f_{n+1}^{\star}(\zeta)+\kappa_{0}^{2}\left[k_{n}^{\star}(\zeta)-f_{n}^{\star}(\zeta)\right]
$$

[1] A. W. Chao, Physics of Collective Beam Instabilities in High Energy Accelerators (Wiley, New York, 1993).

[2] R. L. Gluckstern, R. K. Cooper, and P. J. Channel, Part. Accel. 16, 125 (1985).

[3] G. Decker and J.M. Wang, in Proceedings of the 1987 Particle Accelerator Conference, Washington, DC (IEEE, Piscataway, NJ, 1987), Catalog No. 87CH2387-9, p. 1210.

[4] G. Decker and J. M. Wang, Phys. Rev. D 38, 908 (1988).

[5] K. Yokoya, DESY Technical Report No. 86-084, 1986 (unpublished).

[6] R. L. Gluckstern, F. Neri, and R. K. Cooper, Part. Accel. 23, 53 (1988).

[7] K. A. Thompson and R. D. Ruth, Phys. Rev. D 41, 964 (1990).

[8] F. Neri and R. L. Gluckstern, in Proceedings of the 1989 Particle Accelerator Conference, Chicago, IL (IEEE, Piscataway, NJ, 1989), p. 812.

[9] W. K. H. Panofsky and M. Bander, Rev. Sci. Instrum. 39, 206 (1968).

[10] V. K. Neil and R. K. Cooper, Part. Accel. 1, 111 (1970).

[11] V. K. Neil, L. S. Hall, and R. K. Cooper, Part. Accel. 9, 213 (1979).

[12] Y. Y. Lau, Phys. Rev. Lett. 63, 1141 (1989).

[13] K. Takayama, Phys. Rev. A 45, 1157 (1992).

[14] C.L. Bohn and J.R. Delayen, Phys. Rev. A 45, 5964 (1992).

[15] J. J. Bisognano and R. L. Gluckstern, in Proceedings of the 1987 Particle Accelerator Conference, Washington, DC (Ref. [3]), p. 1078.

[16] G. H. Hoffstaetter and I. V. Bazarov, Phys. Rev. ST Accel. Beams 7, 054401 (2004).

[17] J.R. Delayen, Phys. Rev. ST Accel. Beams 6, 084402 (2003).

[18] J.R. Delayen, Phys. Rev. ST Accel. Beams 7, 074402 (2004).
[19] V.E. Balakin, A. V. Novokhatsky, and V.P. Smirnov, in Proceedings of the 12th International Conference on High-Energy Accelerators, Batavia, IL (Fermilab, Batavia, 1983), p. 119.

[20] G. V. Stupakov, in Proceedings of the XX International LINAC Conference, Monterey, CA, 2000 (SLAC Report No. SLAC-R-561, 2000), p. 272.

[21] D. Chernin and A. Mondelli, Part. Accel. 24, 177 (1989).

[22] R. Gluckstern, F. Neri, and J. B. J. van Zeijts, in Proceedings of the 1990 Linear Accelerator Conference, Albuquerque, NM (LANL Report No. LA-12004-C, 1990), p. 309.

[23] R. L. Gluckstern, J.B. J. van Zeijts, and F. Neri, in Proceedings of the 1991 Particle Accelerator Conference, San Francisco, CA (IEEE, Piscataway, NJ, 1991), Catalog No. 91CH3038-7, p. 1597.

[24] G. V. Stupakov, SLAC Technical Report No. SLAC-AP108, 1997 (unpublished).

[25] D. A. Whittum, J. Phys. A 30, 8751 (1997).

[26] G. Guignard and J. Hagel, in Proceedings of the XIX International LINAC Conference, Chicago, IL, 2000 (ANL Report No. ANL-98/28, 2000), p. 484.

[27] G. Guignard and J. Hagel, Nucl. Instrum. Methods Phys. Res., Sect. A 434, 179 (1999).

[28] C. L. Bohn and K.-Y. Ng, Phys. Rev. Lett. 85, 984 (2000).

[29] C.L. Bohn and K.-Y. Ng, in Proceedings of the $X X$ International LINAC Conference, Monterey, CA, 2000 (Ref. [20]), p. 31.

[30] K. Y. Ng and C.L. Bohn, in Proceedings of the Second Asian Particle Accelerator Conference (APAC'01), Beijing, 2001 (IHEP, Beijing, 2001), p. 372.

[31] J.T. Seeman, F.-J. Decker, R. L. Holtzapple, and W.L. Spence, in Proceedings of the 1993 Particle Accelerator Conference, Washington, DC (IEEE, Piscataway, NJ, 1993), Catalog No. 93CH3279-7, p. 3234. 